\title{
Nonlinear Transfer Entropy to Assess the Neurovascular Coupling in Premature Neonates
}

\author{
Dries Hendrikx ${ }^{1,2}$, Liesbeth Thewissen ${ }^{3,4}$, Anne Smits ${ }^{3,4}$, Gunnar Naulaers ${ }^{3,4}$, \\ Karel Allegaert ${ }^{3,5,6}$, Sabine Van Huffel ${ }^{1,2}$, and Alexander Caicedo ${ }^{1,2}$ \\ ${ }^{1}$ Department of Electrical Engineering (ESAT), STADIUS, KU Leuven, Belgium. \\ ${ }^{2}$ imec, Leuven, Belgium. \\ ${ }^{3}$ Department of Development and Regeneration, KU Leuven, Belgium. \\ ${ }^{4}$ Department of Neonatology, UZ Leuven, Belgium. \\ ${ }^{5}$ Department of Pediatric Surgery and Intensive Care, Erasmus MC-Sophia Children's \\ Hospital, Rotterdam, The Netherlands. \\ ${ }^{6}$ Department of Neonatology, Erasmus MC-Sophia Children's Hospital, Rotterdam, The \\ Netherlands.
}

\begin{abstract}
In the adult brain, it is well known that increases in local neural activity trigger changes in regional blood flow and, thus, changes in cerebral energy metabolism. This regulation mechanism is called neurovascular coupling (NVC). It is not yet clear to what extent this mechanism is present in the premature brain. In this study, we explore the use of transfer entropy (TE) in order to compute the nonlinear coupling between changes in brain function, assessed by means of EEG, and changes in brain oxygenation, assessed by means of near-infrared spectroscopy (NIRS). In a previous study, we measured the coupling between both variables using a linear model to compute TE. The results indicated that changes in brain oxygenation were likely to precede changes in EEG activity. However, using a nonlinear and nonparametric approach to compute TE, the results indicate an opposite directionality of this coupling. The source of the different results provided by the linear and nonlinear TE is unclear and needs further research. In this study, we present the results from a cohort of 21 premature neonates. Results indicate that TE values computed using the nonlinear approach are able to discriminate between neonates with and without brain abnormalities, indicating a less functional NVC in neonates with brain abnormalities.
\end{abstract}

\section{Introduction}

In the adult brain, increases in local neural activity trigger increases in regional blood flow, and thus changes in regional cerebral energy metabolism. We call this regulation mechanism between neural activity and hemodynamics neurovascular coupling (NVC). In practice, brain oxygenation levels can be assessed by means of near-infrared spectroscopy (NIRS), while brain function can be measured by means 
of EEG. In the neonatal brain, however, the presence and extent of the MVC is still unclear. Some neonatal fMRI and NIRS studies have reported adult-like NVC [1] [2], while other studies in both humans and rodent models have reported differences in hemodynamic responses in the early postnatal brain compared to adults [3] [4].

In this paper, we use nonlinear and nonparametric transfer entropy (TE) to compute the coupling between NIRS and EEG; we use this measure to assess the $\mathrm{NVC}$ in the premature brain. TE allows to quantify the directionality of information transfer between NIRS and EEG signals. In a previous study, we measured the coupling between NIRS and EEG using a linear model to compute TE [6]. The results indicated that changes in brain oxygenation were likely to precede changes in amplitude of EEG activity, indicating a NVC response that differs from the response commonly observed in adults. IN this regard, however, it is important to mention that the coupling between neuronal activity and brain hemodynamics incorporates nonlinear effects, indicating that a nonlinear measure (such as the nonlinear TE presented in this paper) is more appropriate for NVC quantification [7].

Finally, we compare the NVC in a cohort of neonates that underwent sedation with propofol. We evaluated the prognostic value of TE in order to detect neonates that presented small brain hemorrhages.

\section{Dataset}

Concomitant measurements of brain oxygenation (brain tissue saturation), measured by means of NIRS (INVOS 5100, Covidien, neonatal probe), and raw EEG (Olympic CFM 6000, Natus) were acquired as part of a study to identify the optimal dose of propofol for procedural sedation in neonates [5]. The NIRS optode was located left frontoparietal and the EEG was measured between the $\mathrm{C} 3$ and $\mathrm{C} 4$ of the 10-20 system. The sampling frequency was equal to $1 \mathrm{~Hz}$ and $100 \mathrm{~Hz}$ for NIRS and EEG, respectively. All recordings were shortened to $6 \mathrm{~h}$, with the start of the $6 \mathrm{~h}$ aligned to the moment of propofol administration.

The EEG signal was preprocessed in order to remove bad electrode contact artifacts (impedance exceeding $10 \mathrm{k} \Omega$ ) and motion artifacts, observed as peaks in the EEG impedance. Due to the different temporal characteristics between the NIRS and EEG signal, we used the running root mean squared (RMS) of the EEG, which is a continuous estimate of EEG power. More specifically, the RMS value was computed using a window length of $5 \mathrm{~s}$ and an overlap of $4 \mathrm{~s}$, producing a new value every second. The window length of $5 \mathrm{~s}$ was defined in order to assess EEG delta activity.

The study group consisted of 21 premature neonates, stratified into two groups based on the presence of brain abnormalities, observed on routine ultrasound scans. More information on the study group is presented in Table 1. 


\section{Methods}

We use TE to assess the NVC. TE measures the amount of information transferred from one signal (process) to another. More specifically, the TE from one signal $X$ to another signal $Y$ is the amount of uncertainty that is reduced in future values of $Y$ by knowing the past values of $X$ given the past values of $Y$. Mathematically, if $X$ and $Y$ denote two discrete-time signals, the TE is defined as:

$$
T_{X \rightarrow Y}=H\left(Y_{n} \mid Y_{n}^{-}\right)-H\left(Y_{n} \mid X_{n}^{-}, Y_{n}^{-}\right)
$$

where $Y_{n}, Y_{n}^{-}=Y_{n-1: n-L}, X_{n}^{-}=X_{n-1: n-L}$ denote the signal $Y$ at time $n$, the past of $Y$ and the past of $X$, respectively, and $H$ denotes the conditional Shannon entropy, which is defined as

$$
H\left(Y_{n} \mid Y_{n}^{-}\right)=-\sum_{i, j} p\left(y_{i}, \boldsymbol{y}_{j}^{-}\right) \log \frac{p\left(y_{i}, \boldsymbol{y}_{j}^{-}\right)}{p\left(\boldsymbol{y}_{j}^{-}\right)}
$$

where $p\left(y_{i}, \boldsymbol{y}_{j}^{-}\right)$is the probability that $Y_{n}=y_{i}$ and $Y_{n}^{-}=\boldsymbol{y}_{j}^{-}$.

In the present analysis, the TE is estimated by binning the original time series, which allows us to estimate the probability density functions (PDF) required to compute the TE. This approach is very general, since no model is assumed for the data. Therefore, this approach also takes into account nonlinear interactions. Moreover, uniform embedding with dimension $L=10$ was used to capture the past of the signals. In order to assess the statistical significance $(p<0.05)$ of the TE values, we used surrogate data, obtained by randomly shuffling the original signals. Since the TE is a non-symmetric measure, two different thresholds were defined. The TE curves are computed using a window of $15 \mathrm{~min}$ with an overlap of $10 \mathrm{~min}$, producing a new value every $5 \mathrm{~min}$.

Two features are computed from the resulting TE curves in order to quantify the effect of propofol on the TE curves: the minimal value $M$ and the maximal deviation $\Delta$ from baseline. These feature values are computed in the first 3 hours of the analysis window. Baseline level is defined as the median value of the last 3 hours of the analysis window.

\section{Results}

In Figure 1, the median TE curves are presented for the cohort of neonates without (solid circles) and with brain injury (open circles. Significant information flows were determined by Monte Carlo surrogates, i.e., temporally shuffled time series, which leads to the significance thresholds depicted in Figure 1 [10]. The Wilcoxon 
rank-sum test indicates that $T_{\mathrm{EEG} \rightarrow \mathrm{NIRS}}$ is significantly higher than $T_{\mathrm{NIRS} \rightarrow \mathrm{EEG}}(p<$ 0.001).

Figure 2 shows a comparison between the group of neonates without (white boxplots) and with (colored boxplots) brain injuries based on the features $M$ and $\Delta$. In addition, the baseline level is also compared between the two groups. Resulting $p$-values are equal to $0.259,0.845$ and 0.039 for features $M, \Delta$ and the baseline, respectively, which indicates that the response to propofol is comparable in both groups (features $M$ and $\Delta$ ), while background NVC (baseline) is lower in neonates with brain injury.

\section{Discussion and conclusions}

In this study, we found that the transfer of information, quantified by means of TE, is larger in the direction from EEG to NIRS than from NIRS to EEG. This indicates that changes in EEG amplitude are likely to precede changes in NIRS. In other words: changes in brain function trigger changes in brain hemodynamics and metabolism, which is in agreement with the NVC of the adult brain. It is however important to note that the transfer of information NIRS to EEG is not zero, indicating a flow of information also in this direction. This might be caused by the feedback mechanisms that are in charge of the regulation of brain metabolism, i.e. the delivery of nutrients and oxygen to meet the metabolic demand.

In a previous study, we measured the coupling between NIRS and EEG using a linear model to compute TE [6]. The results indicated that changes in brain oxygenation were likely to precede changes in amplitude of EEG activity. This contradicts the results we obtained in the present study. The non-parametric approach to compute TE, used in this study, estimates all the transfer of information from one process to another, including the linear and non-linear part. Taking this into account, and the results presented in [6], we can conclude that the linear model for TE is not able to model the nonlinear processes involved in NVC which, once taken into account, determine a different directionality in the flow of information. In addition, the nonlinear TE is able to capture the effect of propofol in the NVC, which was not observed using the linear model. These results indicate that it is possible that there are two different mechanisms affecting NVC, one acting on its linear part, and another one explained more by a non-linear process.

In Figure 1, lowered values of EEG to NIRS coupling are observed after the administration of propofol, which consequently increase to a steady baseline value in all patients in the study group. Propofol induces a reversible state of diminished responsiveness behaviorally similar to quiet sleep, which is characterized by a more discontinuous EEG and is associated with lower fractional tissue oxygenation extraction (FTOE) values. This indicates lower brain oxygen consumption, which could explain the reduced EEG to NIRS interaction. Based on visual inspection of Figure 1, it appears that the reduction in EEG to NIRS coupling is more severe for 
the cohort of abnormal neonates. A more in-depth study, based on the feature values of $M$ and $\Delta$, presented in Figure 2, indicates that there is no significant difference in response between the neonates without and the neonates with brain injury. The NIRS to EEG coupling is not influenced by propofol.

The TE baseline values, presented in Figure 2, were observed to be significantly lower in the cohort of neonates with brain injury. A lower TE indicates a less functional NVC. Thus, the cerebral vascular network in the neonates with brain injury is less able to compensate for increased metabolism resulting from neuronal activation. These results are in agreement with a study from Mahmoudzadeh et al., who observed a less functional NVC in preterm neonates with intraventricular hemorrhage (IVH) compared to healthy neonates [8]. In addition, Chalak et al. observed a significantly lower NVC in newborns with neonatal encephalopathy (NE) compared to a healthy control group, assessed by means of wavelet coherence [9]. These studies point out that brain abnormalities alter the NVC, and that this alteration in $\mathrm{NVC}$ can be captured using signal processing techniques combining NIRS and EEG measurements. Therefore, we have shown that the nonlinear TE can be used as a physiological marker to guide therapeutic decision-making or predict neurological outcome. However, further studies are needed in order to validate this methodology in a larger cohort of neonates, using a different clinical model.

Acknowledgments Dries Hendrikx is a $\mathrm{SB} \mathrm{PhD}$ fellow at the Fonds voor Wetenschappelijk Onderzoek (FWO) Vlaanderen. This research was also supported by Bijzonder Onderzoeksfonds KU Leuven (BOF): SPARKLE - Sensor-based Platform for the Accurate and Remote monitoring of Kinematics Linked to E-health \#: IDO-13-0358, The effect of perinatal stress on the later outcome in preterm babies \#: C24/15/036 and TARGID - Development of a novel diagnostic medical device to assess gastric motility \#: C32-16-00364. Fonds voor Wetenschappelijk Onderzoek-Vlaanderen (FWO): Hercules Foundation (AKUL 043) 'Flanders BCI Lab - HighEnd, Modular EEG Equipment for Brain Computer Interfacing'. Agentschap Innoveren en Ondernemen (IWT): O\&O HBC 20160184 eWatch. imec funds 2017. imec ICO projects: ICON HBC.2016.0167, 'SeizeIt'. Belgian Foreign Affairs-Development Cooperation: VLIR UOS programs (2013-2019). EU: European Union's Seventh Framework Programme (FP7/2007-2013): The HIP Trial: \#260777. ERAMUS+: INGDIVS 2016-1 SE01-KA203-022114. European Research Council: The research leading to these results has received funding from the European Research Council under the European Union's Seventh Framework Programme (FP7/2007-2013) / ERC Advanced Grant: BIOTENSORS (no 339804). This paper reflects only the author's views and the Union is not liable for any use that may be made of the contained information. EU H2020FETOPEN 'AMPHORA' \#766456.

\section{References}

1. Arichi T, Moraux A, Melendez A et al. (2010), "Somatosensory cortical activation identified by functional MRI in preterm and term infants," Neuroimage, 49:2063-71.

2. Liao S, Gregg N, White B et al. (2010), "Neonatal hemodynamic response to visual cortex activity: high-density near-infrared spectroscopy study," J Biomed Opt, 15:1-9. 
3. Kozberg M, Chen B, Deleo S et al. (2013), "Resolving the transition from negative to positive blood oxygen level-dependent responses in the developing brain," Proc Natl Acad Sci, 110:4380-5

4. Zehendner C, Tsohataridis S, Luhmann H et al. (2013), "Developmental switch in neurovascular coupling in the immature rodent barrel cortex," PLoS One, 8:e80749.

5. Smits A, Thewissen L, Caicedo A et al. (2016), "Propofol Dose-Finding to Reach Optimal Effect for (Semi-)Elective Intubation in Neonates," J Pediatr, 179:54-60.

6. Caicedo A, Thewissen L, Smits A et al. (2016), "Changes in oxygenation levels precede changes in amplitude of the EEG in premature infants," Adv Exp Med Biol, 923:143-149.

7. Sheth SA, Nemoto M, Guiou M et al. (2004), "Linear and Nonlinear Relationschips between Neuronal Activity, Oxygen Metabolism, and Hemodynamic Responses", Neuron, 42:347-355.

8. Mahmoudzadeh M, Dehaene-Lambertz G, Kongolo G et al. (2018), "Consequence of intraventricular hemorrhage on neurovascular coupling evoked by speech syllables in preterm neonates," Dev. Cog. Neurosc., 30:60-69.

9. Chalak L, Fenghua T, Beverley A-H et al. (2017), "Novel Wavelet Real Time Analysis of Neurovascular Coupling in Neonatal Encephalopathy," Scientific Reports, 7:45958.

10. Ruddell BL and Kumar P (2009), "Ecohydrologic process networks: 1. identification", Water Resources Research, 45:W03419. 
Table 1. Stratification of the original study group into two subgroups, based on the presence of brain abnormalities on a routine ultrasound scan. For each group characteristic, the median value is presented, together with the interquartile range (in brackets). The $p$-value was computed using the Wilcoxon rank-sum test.

\begin{tabular}{|l|l|l|l|}
\hline & \multicolumn{2}{|c|}{ Population } & \\
\hline & Normal $(n=15)$ & Abnormal $(n=6)$ & $p$-value \\
\hline Postmenstrual age (weeks) & $30(29-30.5)$ & $29.5(28.25-30)$ & 0.5471 \\
\hline Postnatal age (days) & $1(1-1)$ & $1(1-1.75)$ & 0.1247 \\
\hline Propofol dose (mg/kg) & $1(1-1.50)$ & $1(0.625-1.75)$ & 0.6285 \\
\hline
\end{tabular}

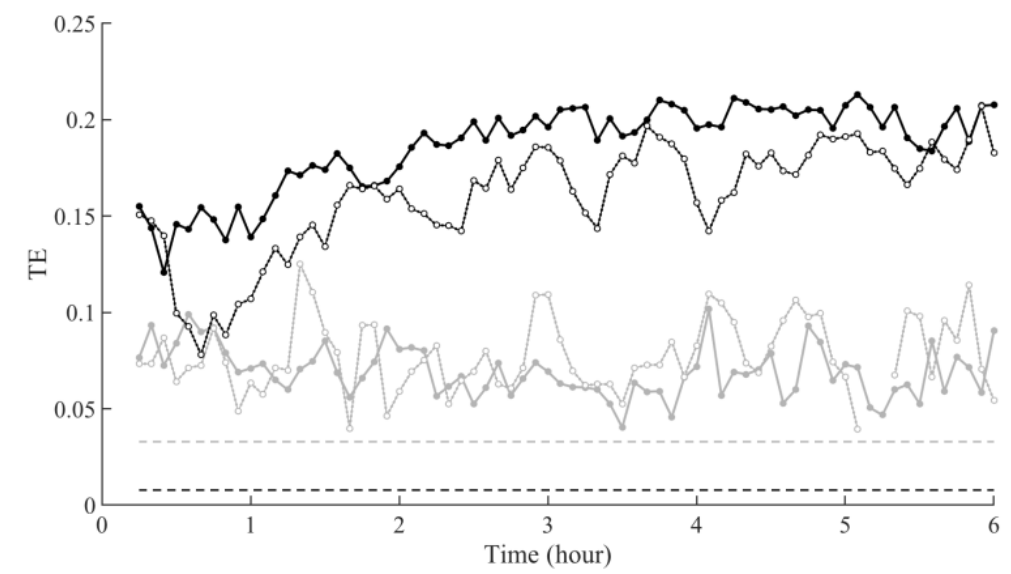

Fig. 1. TE curves after administration of propofol at $t=0 ; T_{\mathrm{NIRS} \rightarrow \mathrm{EEG}}$ and $T_{\mathrm{EEG} \rightarrow \mathrm{NIRS}}$ are depicted in gray and black, respectively. The solid circles represent the median values for normal neonates, while the open circles indicate the abnormal group. The dashed lines indicate the significance levels $(p<0.05)$ for the TE values, obtained by surrogate data analysis. 

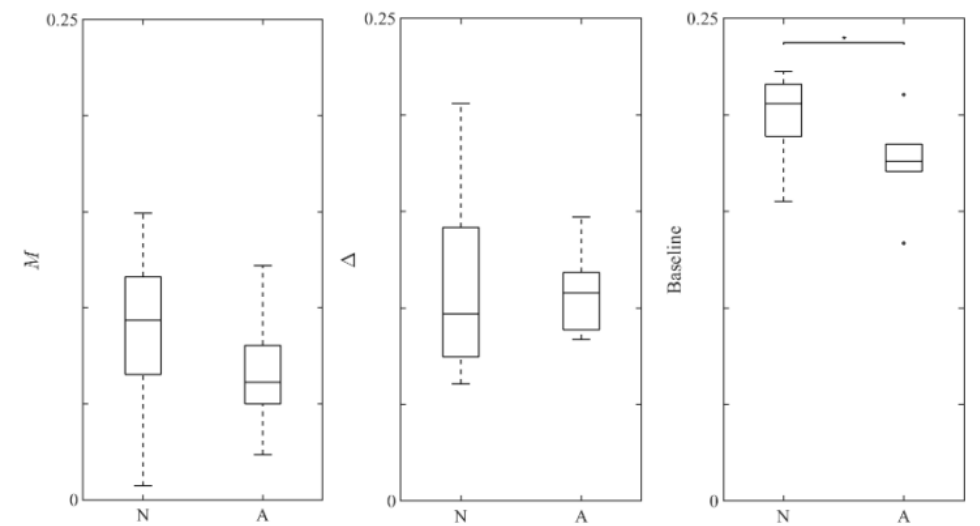

Fig. 2. Comparison of feature and baseline values between the normal and abnormal neonates; $M$, $\Delta$, and baseline indicate the minimal value, the maximal deviation from baseline and baseline values, respectively. 